\title{
Investigation of Water Sampling Assisted by Jets for Active Chemical Sensing System
}

\author{
Hanako Ishida $^{1}$, Tatsuki Mitsuishi ${ }^{1}$, Haruka Matsukura ${ }^{2}$, Hiroshi Ishida $^{1}$ \\ ${ }^{1}$ Tokyo University of Agriculture and Technology, Tokyo, Japan, \\ 2 Osaka University, Osaka, Japan \\ h_ishida@cc.tuat.ac.jp
}

\begin{abstract}
:
Here we report our investigations on the effectiveness of jet-assisted active chemical sampling. Crayfish generate jets by waving maxillipeds. The flow induced by the jets is considered to help collect odor samples to their olfactory organs. In theory, the velocity decay of such jet-induced flow with the distance to a jet is much smaller than the velocity decay of the flow generated by sucking water using a pump. Results of experiments and flow simulations show that water samples are drawn from the surroundings to a jet discharge device in much shorter time than by a water suction device. The obtained insights can be used to enable highly sensitive chemical detection in underwater robots.
\end{abstract}

Key words: active sensing, chemical sensor, jet entrainment, crayfish, olfactory search behavior

\section{Introduction}

A crayfish has three pairs of maxillipeds around its mouth part below the antennules, and the flagella of these maxillipeds have a fan-like shape. As shown in Fig. 1, jet-like water currents are generated to the sides by waving maxillipeds. Since the jets entrain the surrounding water, an inflow to the jets is induced [1]. It has been suggested that odor sample collection from the surroundings to the antennules is promoted by this inflow. Moreover, crayfish can change the directions of the jet discharge, and are known to generate at least several different flow fields around them [1].

Such jet-assisted water sampling can be used to enable highly sensitive chemical sensing on underwater robot platforms. In water, it is especially important to collect chemical substances onto chemical sensors on board the robots because the molecular diffusion in water is extremely slow. Some deep-sea research vessels are equipped with a pump to suck water samples for chemical analysis. When water is sucked into a small inlet port, a centrosymmetric flow field converging to the port is generated. The velocity of this flow decays rapidly with the distance from the inlet port; the velocity is proportional to the inverse square of the distance [2]. On the other hand, axisymmetric flow is induced by a jet, and its velocity is proportional to the inverse of the distance to the jet. Therefore, the velocity decay is much smaller [2].

In our previous work [1], changes in the angular range of the chemical sample collection with the jet discharge directions were investigated. Here we report the results of computational fluid dynamics (CFD) simulations conducted to investigate the time required to collect water samples. The results complement the experimental investigation reported in [2].

\section{Experiments}

A $60 \mathrm{~mm}$ long jet discharge device was used to model the jet generation by crayfish [1] and compare jet-assisted water sample collection with simple suction. Water is sucked by pumps from an inlet port on the front side of the device at $300 \mathrm{~mL} / \mathrm{min}$, and then discharged from two outlet ports on the sides. The device was

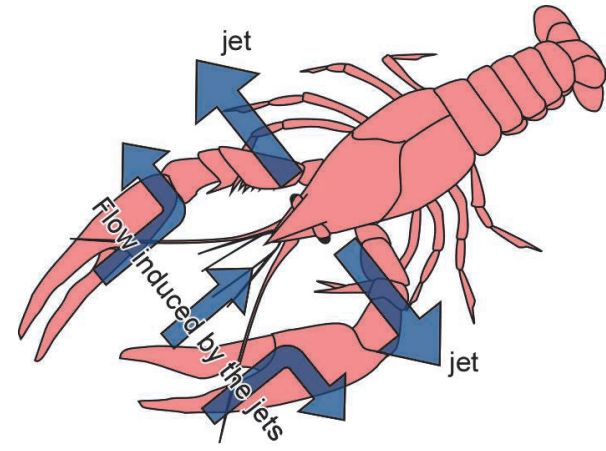

Fig. 1. Flow induced by the jets. 
placed in the middle of a water container (1200 $\mathrm{mm}$ long and $800 \mathrm{~mm}$ wide) which was filled with water to a depth of $100 \mathrm{~mm}$. A line of tubes was placed at $60 \mathrm{~mm}$ from the device, and aqueous solution of a fluorescent ink (Rhodamine 6G) was released from these tubes to visualize the flow field generated by the jet discharge device. The time required to collect water samples from the surroundings was measured by visually observing the development of the streaklines of the ink.

As shown in Table 1, the time required to draw ink blobs for the same pumping speed was significantly reduced by using jets. The time required to draw water samples was shorter when the jets were discharged to the sides than when discharged $45^{\circ}$ backward.

\section{CFD Simulations}

In the experiments, jets generated by the device collided with the walls of the water container. A part of the jets deflected by the walls induce circulating flow in the water container. The short water sample collection time in the experiments might be the results assisted by this circulating flow. Therefore, CFD simulations were conducted.

A commercial CFD software package, ANSYS Fluent 14.5, was used to calculate the flow fields. The realizable $k-\varepsilon$ model was chosen as a turbulence model. The no-slip boundary conditions were applied to the bottom of the water container and the walls of the jet discharge device. The free-slip conditions were applied to the water surface. To the lateral sides of calculation domain, the open boundary conditions were applied. Fig. 2(a) shows the flow field calculated by the CFD simulation when jets were discharged to the sides. In this flow field, water samples are drawn straight to the jets. Fig. 2(b) shows the flow field calculated by the CFD simulation when jets were discharged $45^{\circ}$ backward. It can be seen that water samples are converging to the device.

The time required to draw water samples from $60 \mathrm{~mm}$ to $10 \mathrm{~mm}$ was calculated by using the flow velocity distribution. While a similar value, $24.0 \mathrm{~s}$, was obtained for the jet discharge at $45^{\circ}$ backward, the calculated time for the jet discharge to the sides, $26.9 \mathrm{~s}$, was significantly longer than the time obtained in the experiments. In contrast to the experimental results, it was found that water samples can be collected in a shorter time by discharging jets $45^{\circ}$ backward when there is no wall around.

\section{Conclusion}

Water sample collection time is significantly reduced by using the jets. The present results
Tab. 1: Time required to draw ink blobs from distance of $60 \mathrm{~mm}$ to $10 \mathrm{~mm}$ in front of the jet discharge device.

\begin{tabular}{ccc}
\hline $\begin{array}{c}\text { Simple } \\
\text { suction }\end{array}$ & $\begin{array}{c}\text { Jet discharge } \\
\text { to sides }\end{array}$ & $\begin{array}{c}\text { Jet discharge at } \\
45^{\circ} \text { backward }\end{array}$ \\
\hline $104.9 \mathrm{~s}$ & $18.2 \mathrm{~s}$ & $29.5 \mathrm{~s}$ \\
\hline
\end{tabular}

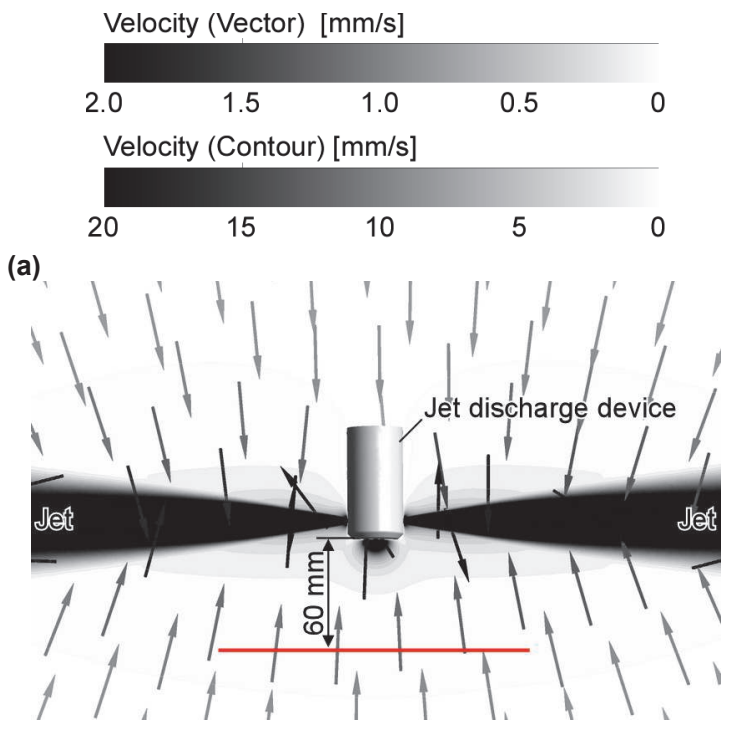

(b)

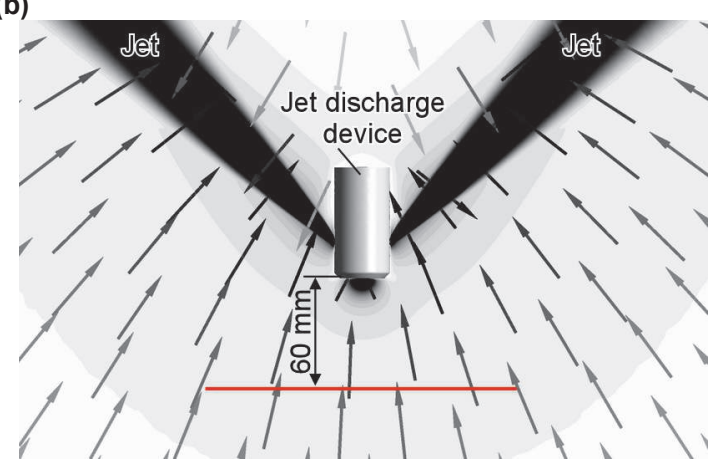

Fig. 2. Results of CFD simulations. (a) Jet discharge to sides. (b) Jet discharge at $45^{\circ}$ backward. Vector field is overlaid on the velocity contour shown in gray scale.

suggest that underwater robots can collect more chemical samples in a given time by discharging the sucked water at $45^{\circ}$ backward.

\section{References}

[1] R. Takemura, K. Takahashi, T. Makishita, H. Ishida, Adaptive Chemical Sampling Device Inspired by Crayfish, ECS Trans. 50, 259-266 (2012); doi: 10.1149/05012.0259ecst

[2] H. Ishida, T. Mitsuishi, R. Takemura, H. Matsukura, H. Ishida, Chemical Sampling Device for Underwater Robot: Jet Discharge Mimicking crayfish, Proc. ISOCS/IEEE Int. Symp. Olfaction and Electronic Nose, 116-118 (2017); doi: 10.1109/ISOEN.2017.7968882 